\title{
Role of Histopathological Correlation in Cases of Sino-Nasal Masses: A Retrospective Analysis
}

\section{Ayushi Gupta ${ }^{1}$, Sahil Kapoor ${ }^{2 *}$, Poonam K Saidha ${ }^{3}$, Pooja Das ${ }^{1}$ and Arun Kumar ${ }^{4}$}

${ }^{1}$ Junior Resident, Department of ENT, FMHS, SGT University, Gurugram, Haryana, India

${ }^{2}$ Assistant Professor, Department of ENT, FMHS, SGT University, Gurugram, Haryana, India

${ }^{3}$ Associate Professor, Department of ENT, FMHS, SGT University, Gurugram, Haryana, India

${ }^{4}$ Professor, Department of ENT, FMHS, SGT University, Gurugram, Haryana, India

*Corresponding Author: Sahil Kapoor, Assistant Professor, Department of ENT, FMHS, SGT University, Gurugram, Haryana, India.
Received: July 29, 2021

Published: August 09, 2021

(C) All rights are reserved by Sahil Kapoor., et al.

\begin{abstract}
Objectives: Sinonasal masses affect people of all age groups and cause significant impairment of quality of life. The present study aimed to assess the clinico-pathological profile of patients presenting with sino-nasal masses and emphasize the role and importance of pre-operative histopathology of their sino-nasal masses.

Methods: Fifty five patients (10 - 68 years age) presenting to a tertiary care centre in Gurugram, Haryana with sino-nasal masses over the course of 3 years were included in a retrospective analysis. All masses were sent for histopathological examination for final diagnosis prior to surgical intervention.

Results: The most common presenting complaint was nasal obstruction (38\%). Ninety percent of sino-nasal masses were found to be benign, of which, nasal polyp (40\%) was the most common biopsy finding. Amongst malignant lesions, squamous cell carcinoma $(60 \%)$ was the most common histological type.

Conclusion: Correlation of clinical and histopathological finding is of utmost importance for accurate and timely diagnosis of sinonasal masses. It will enable prompt and better management of these patients, which will decrease morbidity and mortality associated with sino-nasal masses.
\end{abstract}

Keywords: Sinonasal Mass; Histopathology; Nasal Polyps; FESS

\section{Introduction}

Mass in the nasal cavity, paranasal sinuses (PNS) and nasopharynx is a common presentation in ENT (Ear, Nose and Throat) practice. Patients of all age groups present with sino-nasal masses. Sino-nasal masses lead to a significant decrease in the quality of life of patients. Sino-nasal masses can be classified as congenital, inflammatory, neoplastic (benign or malignant) and traumatic [1]. Majority of them are inflammatory in nature, with neoplasms comprising approximately $3 \%$ of all head and neck tumors [2]. Patients with sino-nasal masses, whether bilateral or unilateral, pres- 
ent with various symptoms, such as nasal obstruction, rhinorrhea, blood-stained nasal discharge, epistaxis, oral symptoms, facial swelling, orbital symptoms and ear-related symptoms [3].

A variety of neoplastic and non-neoplastic sinonasal lesions can present as nasal polyps. Most nasal polyps are a consequence of chronic inflammation of the epithelium of nasal mucosa and paranasal sinuses, as a result of allergy and infection [4]. They often present as unilateral or bilateral pedunculated, soft, smooth, translucent and round or pear-shaped masses of inflamed mucosa prolapsing into the nose. Polyps are a common cause of nasal obstruction in adults and have a prevalence of 4\% [5,6]. Unilateral nasal masses are rare and are more likely to be malignant or premalignant, whereas bilateral masses are inflammatory in nature. Amongst unilateral nasal masses, antrochoanal polyp, fungal ball, chronic sinusitis with polypoidal changes, inverted papilloma, angiofibroma, nasal carcinoma or sarcoma type tumour are seen [7].

Clinical diagnosis in such cases is based on a detailed history and examination of the nose and PNS. Often, other diagnostic tests (nasal endoscopy, radiology, allergy tests and histopathological examination (HPE)) are required, especially, in cases of unilateral nasal masses to reach the final diagnosis. In a series of sinonasal polyp cases, $2.3 \%$ showed neoplastic changes on histopathology. This highlights the fact that despite low incidence, it is prudent to conduct HPE for early diagnosis and treatment of life threatening sino-nasal conditions $[7,8]$.

\section{Aim and Objective of the Study}

1. To study the clinical presentation of various sinonasal masses.

2. To evaluate the histopathological profile of the sinonasal masses.

3. To emphasize the role and importance of pre-operative histopathology in cases of sinonasal masses.

\section{Materials and Methods}

A retrospective analysis of 55 patients was carried out in Department of Otorhinolaryngology at a tertiary care centre in Haryana after taking institutional ethical committee approval (SEC/ FMHS/MD/16/06/21-70). All patients diagnosed with sinonasal masses in the age group of 10 - 68 years in past 3 years were included in this study. Detailed history and a complete ENT examina- tion were done of all patients, including anterior rhinoscopy, posterior rhinoscopy and diagnostic nasal endoscopy. On the basis of intra-operative findings, the patients underwent different surgical procedures such as Endoscopic Sinus Surgery (ESS), Endoscopic Excision, Turbinectomy or Caldwell Luc procedure with surgical debridement. All sino-nasal specimens were sent for histopathological examination and a final diagnosis was made.

Statistical analysis was done using Microsoft excel and SPSS version 24 .

\section{Results}

In the present study, 55 cases of sino-nasal masses were analysed, which were from 36 males and 19 females (10 - 68 years age), as shown in table 1.

\begin{tabular}{|c|c|c|}
\hline \multicolumn{3}{|c|}{ Demographic Data } \\
\hline Gender distribution & $\mathbf{N}$ & $\mathbf{\%}$ \\
\hline Male & 36 & $65 \%$ \\
\hline Female & 19 & $35 \%$ \\
\hline Age in years & & \\
\hline $\begin{array}{c}\text { Minimum-maximum } \\
\text { Mean } \pm \text { SD }\end{array}$ & $\begin{array}{c}|c| \\
37.45 \pm 13.23\end{array}$ \\
\hline
\end{tabular}

Table 1: Demographic data of the study population $(n=55)$.

As shown in figure 1, the most common presenting complaint in the study population was nasal obstruction (38\%) followed by nasal discharge $(22 \%)$ and nasal mass $(18 \%)$.

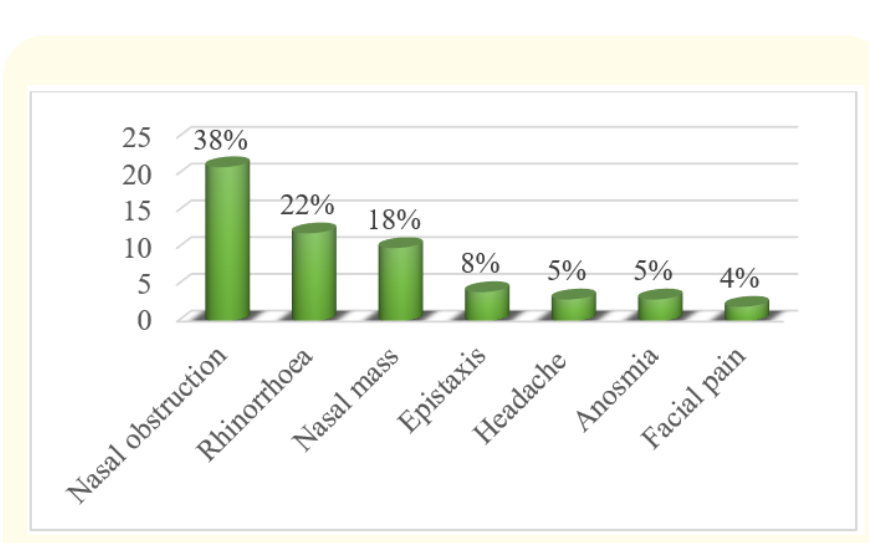

Figure 1: Presenting symptoms among the study population $(n=55)$ 
On diagnostic nasal endoscopy (DNE), fleshy nasal polyposis was the most common clinical finding ( $\mathrm{n}=23,42 \%)$ followed by inferior turbinate hypertrophy $(n=13,24 \%)$ and necrotic masses ( $\mathrm{n}=7,13 \%)$ in the nasal cavity.

Table 2 shows detailed clinical and post-operative histopathological findings of the study population.

\begin{tabular}{|c|c|c|}
\hline DNE finding & $\mathbf{N}$ & $\%$ \\
\hline Nasal polyp $(\mathrm{B} / \mathrm{L}=12, \mathrm{U} / \mathrm{L}=11)$ & 23 & $42 \%$ \\
\hline Nasal cavity mass & 6 & $11 \%$ \\
\hline Inferior turbinate hypertrophy & 13 & $24 \%$ \\
\hline Septal mass & 3 & $5 \%$ \\
\hline Nasopharyngeal mass & 3 & $5 \%$ \\
\hline Necrotic mass & 7 & $13 \%$ \\
\hline Histopathological Finding & $\mathbf{N}$ & $\%$ \\
\hline Nasal polyp $(\mathrm{B} / \mathrm{L}=12, \mathrm{U} / \mathrm{L}=8)$ & 20 & $36 \%$ \\
\hline Inferior turbinate $(B / L=8, U / L=5)$ & 13 & $24 \%$ \\
\hline Inverted papilloma & 3 & $5 \%$ \\
\hline Schwannoma & 1 & $2 \%$ \\
\hline Angiofibroma & 2 & $3 \%$ \\
\hline Squamous cell carcinoma & 3 & $5 \%$ \\
\hline Nasopharyngeal carcinoma & 1 & $2 \%$ \\
\hline Haemangioma & 1 & $2 \%$ \\
\hline Mucous cyst & 1 & $2 \%$ \\
\hline Non-Hodgkin's lymphoma & 1 & $2 \%$ \\
\hline Rhinosporidiosis & 1 & $2 \%$ \\
\hline Rhinoscleroma & 1 & $2 \%$ \\
\hline Rhinolith & 1 & $2 \%$ \\
\hline Mucor mycosis & 5 & $9 \%$ \\
\hline Osteoma & 1 & $2 \%$ \\
\hline Surgical procedure & $\mathbf{N}$ & $\%$ \\
\hline FESS & 27 & $49 \%$ \\
\hline Caldwell-Luc with Endoscopic debridement & 4 & $7 \%$ \\
\hline Endoscopic excision & 11 & $20 \%$ \\
\hline Turbinectomy & 13 & $24 \%$ \\
\hline
\end{tabular}

Table 2: Detailed clinical findings by DNE, histopathological findings and surgical procedure done.
Figure $2 \mathrm{~A}$ and $2 \mathrm{~B}$ illustrate the disease spectrum among patients with benign (90\%) and malignant (10\%) conditions on the basis of histopathological examination.

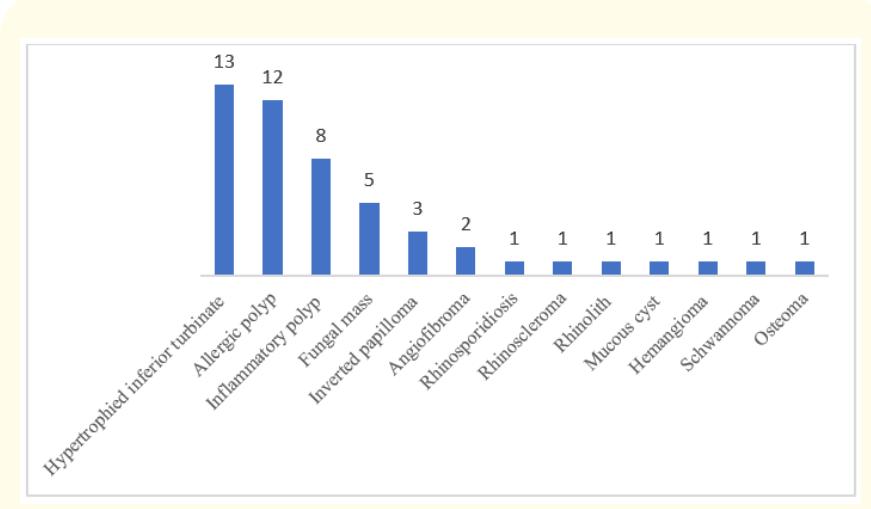

Figure 2A: Disease spectrum of Benign sinonasal masses in our study

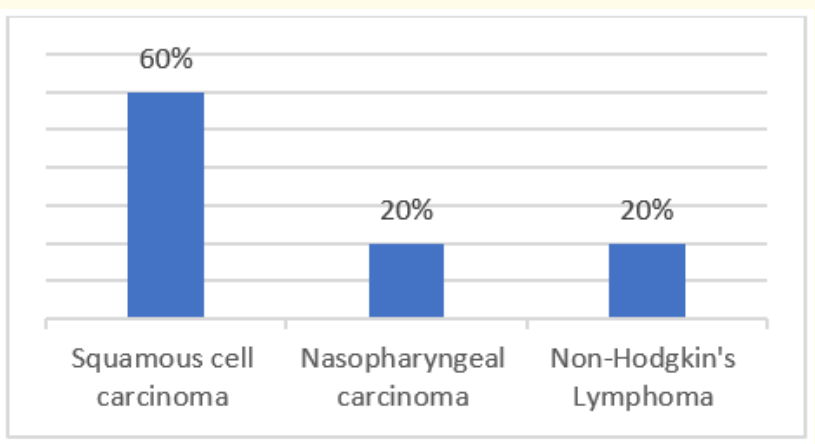

Figure 2B: Disease spectrum of Malignant sinonasal masses in our study

Most nasal polyposis $(n=23)$ in patients were reported to be allergic (ethmoidal polyp, $\mathrm{n}=12$ ) or inflammatory (antrochoanal polyp, $n=8$ ) in nature, while others were found to be angiofibroma $(n=1)$, mucous cyst $(n=1)$ and rhinosporidiosis $(n=1)$ on histopathology. Five necrotic masses showed fungal growth on $\mathrm{KOH}$ (Potassium Hydroxide) mount which was suggestive of mucormycosis, for which, ESS with debridement was done. The other 2 patients with necrotic masses (rhinoscleroma $(\mathrm{n}=1)$ and rhinolith ( $\mathrm{n}$ $=1$ )) underwent endoscopic excision of their lesions. 
Five cases that presented as nasal masses on clinical examination were diagnosed with Schwannoma $(\mathrm{n}=1)$, Squamous cell carcinoma ( $\mathrm{n}=2$ ), Osteoma ( $\mathrm{n}=1$ ) and Non-Hodgkin's Lymphoma ( = 1) after histopathological examination. Three cases of inverted papilloma were reported, out of which, 2 involved the nasal cavity and 1 arose from the nasal septum. One case of septal haematoma was also reported.

Nasopharyngeal masses $(n=3)$ after excision were reported as angiofibroma $(n=1)$, Squamous cell carcinoma $(n=1)$ and Nasopharyngeal carcinoma $(n=1)$ on histopathology.

On the basis of clinical diagnosis, 27 patients (49\%) underwent ESS, 13 patients (24\%) underwent Turbinectomy, and 11 patients underwent Endoscopic excision of nasal mass, followed by Caldwell-Luc with Endoscopic debridement in 4 patients (7\%) (Figure 3).

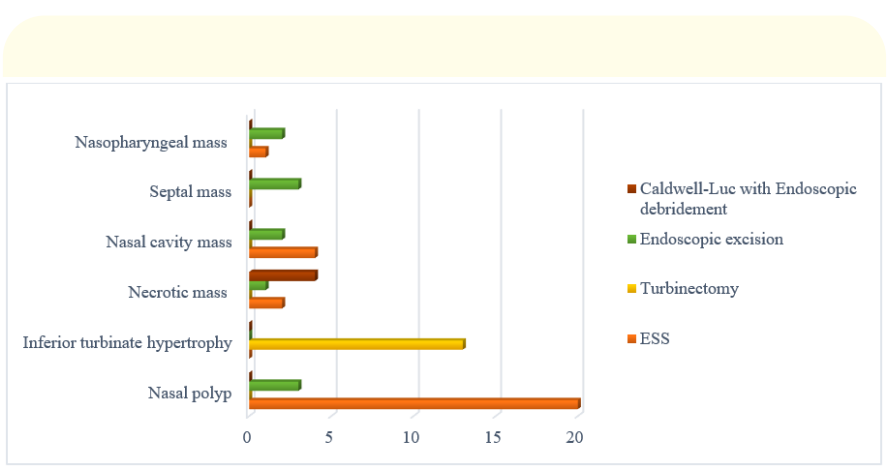

Figure 3: Distribution of patients with DNE findings versus surgical intervention $(n=55)$.

\section{Discussion}

Many benign and malignant conditions present as a mass in the nasal cavity, paranasal sinuses and/or nasopharynx. Clinically, a diagnosis of nasal polyposis is made. In our study, we observed that males (65\%) were more commonly affected than females (35\%). Swami., et al. showed a similar sex distribution (66\% males and $34 \%$ females) in their study [2]. In the present study, age of the participants ranged from 10 - 68 years (Mean = 37.45; SD = 13.23), whereas Zafar., et al. reported a mean age of 22.5 years in their study [9].

Similar to findings of Aljafar., et al. nasal obstruction (38\%) was the most common presenting symptom in patients with sino-nasal masses [10]. Other common presenting symptoms were nasal discharge (22\%) and nasal mass (18\%). Similar observation was made in a study $(\mathrm{n}=50)$ by Bhargava, with $42 \%$ cases presenting with nasal obstruction, 22\% with nasal discharge and 14\% with nasal mass [5].

Most sino-nasal masses in our study were nasal polyposis $(\mathrm{n}=$ $23,42 \%$ ) on DNE, of which 12 were bilateral and 11 were unilateral. The other findings were inferior turbinate hypertrophy (24\%), necrotic nasal mass (13\%), nasal cavity mass (9\%), septal mass (7\%) and nasopharyngeal mass (5\%). Akin to our study, Kumar., et al. also observed $42 \%$ cases of nasal polyp and $17 \%$ cases of turbinate hypertrophy [11].

Sino-nasal diseases can be categorized as benign and malignant [10]. We observed $90 \%$ of sino-nasal masses to be benign with nasal polyps (40\%) being the most common followed by hypertrophied turbinates (26\%), mucormycosis (10\%), inverted papilloma $(6 \%)$ and angiofibroma (4\%). Sino-nasal lesions were found to be more common in studies conducted by Aljafar., et al., Lathi., et al. and Bhargava $[1,5,10]$.

A strong association of nasal polyps is found with allergy, infection, asthma and aspirin sensitivity [1,2,4]. Ethmoidal polyps have been found to be allergic in nature whereas antrochoanal polyps are inflammatory. In our study, the incidence of ethmoidal polyps and antrochoanal polyps was $60 \%$ and $40 \%$, respectively. Lathi., et al. observed $44.6 \%$ ethmoidal polyps (allergic) and $17.8 \%$ antrochoanal polyps (inflammatory) in their study [1].

Inverted papilloma is a rare benign sino-nasal condition notorious for its potential for malignant transformation [1]. While we report a $5 \%$ incidence of inverted papilloma, other researchers have reported a higher incidence: $42.59 \%$ [12], 36.8\% [1] and $30.7 \%$ [13].

Incidence of angiofibroma (3\%) in our study was found to approximate the findings of Lathi., et al. (2\%) and Bhargava (3.4\%) but differed significantly from Richa., et al. (12.4\%) [1,5,4].

Mucormycosis is an aggressive and fatal fungal infection, commonly seen in immunocompromised individuals. It commonly affects the oronasal cavity or paranasal sinuses and may present as a necrotic sino-nasal mass $[14,15]$. In our study, 5 necrotic masses showed fungal growth suggestive of mucormycosis on $\mathrm{KOH}$ mount.

Rhinosporidiosis is an endemic disease in India, Sri Lanka and some African nations [16], while Rhinoscleroma is endemic to north-western parts of India [1]. Rhinosporidiosis $(\mathrm{n}=1 ; 2 \%)$ and 
Rhinoscleroma ( $n=1 ; 2 \%)$ were rare diagnoses in our study. This finding concurred with Pradhananga., et al. [17] but differed greatly from findings of Agarwal., et al. (Rhinosporidiosis- 20.9\% and Rhinoscleroma- 5.55\%) [13].

Amongst other benign lesions, we found 2\% cases of haemangioma $(\mathrm{n}=1)$ and mucous cyst $(\mathrm{n}=1)$. A similar incidence of mucous cyst $(1.78 \%)$ was reported by Lathi., et al. however, they found a higher incidence of haemangioma (47.3\%) in their study.

Malignant lesions of sino-nasal tract are rare. Amongst them, squamous cell carcinoma is the most common histological type [2]. Squamous cell carcinoma $(n=5)$ accounted for $60 \%$ of all malignant sino-nasal masses in our study. Findings were comparable with that of Ajiya., et al. (50.36\%) and Swami., et al. (40\%) [2,12]. Nasopharyngeal carcinoma (2\%) and Non-Hodgkin's Lymphoma (2\%) were also reported in this study and were in keeping with findings of Aljafar., et al [10].

In the present study, Endoscopic sinus surgery (49\%) was the most common surgical intervention performed, which was followed by turbinectomy (24\%) and endoscopic excision of nasal masses (20\%) (Image 1 and 2).

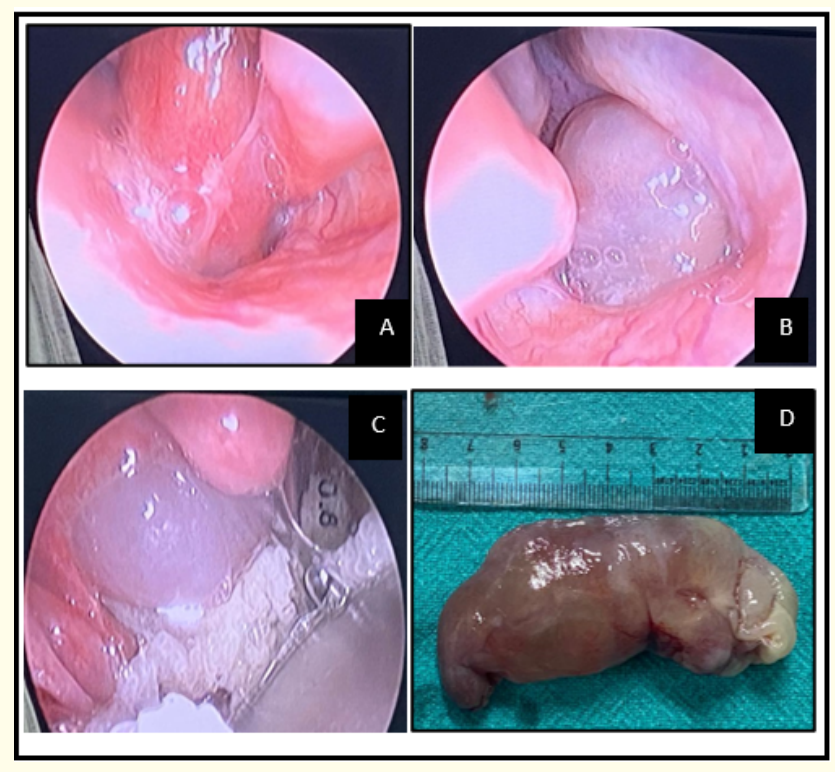

Image 1: Antrochoanal polyp arising from left maxillary sinus into the nasal cavity (A), extending into the posterior choana, nasopharynx (B) and oropharynx (C); 7.6 cm X 3cm Polyp after removal (D)

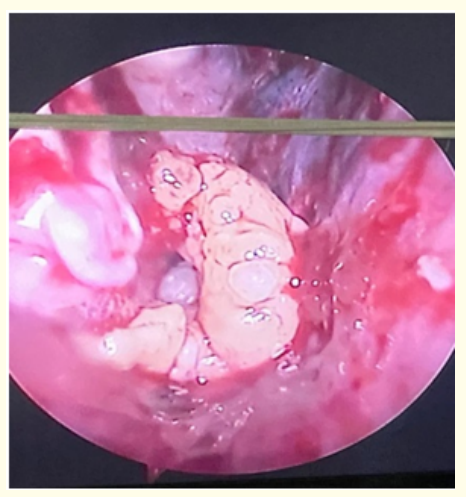

Image 2: Intraoperative picture of sinonasal mass during FESS diagnosed as Rhinosporidiosis on HPE

\section{Conclusion}

A wide spectrum of common and rare diseases encountered in ENT practice present with a sino-nasal mass. Clinical diagnosis is often difficult in such cases. This makes histopathological examination of biopsy specimen necessary for a definitive diagnosis. Malignant masses should be distinguished from benign lesions. Nasal polyp is the most common benign sino-nasal lesion whereas squamous cell carcinoma is the most common malignant sinonasal lesion. Clinico-pathological correlation is of utmost important for accurate and timely diagnosis. Such an approach will enable prompt and better management of these patients, which will decrease morbidity and mortality associated with sino-nasal masses.

The purpose of this study was to emphasize the role of pre-operative biopsy and histopathological examination as an essential part of evaluation of patients with sino-nasal masses, except in cases of angiofibroma, where biopsy is contraindicated. This prudent approach will ensure timely intervention, thereby preventing any future complications.

\section{Bibliography}

1. Lathi M., et al. "Clinico-pathological profile of sinonasal masses: a study from a tertiary care hospital of India". ACTA Otorhinolaryngologica Italica 31 (2011): 372-377.

2. Swami S., et al. "Clinicopathological study of non-neoplastic and neoplastic sinonasal lesions". Journal of Diagnostic Pathology and Oncology 2.2 (2017): 40-43. 
3. Somani S., et al. "Mischievous presentation of nasal masses in rural areas". Asian Journal of Ear, Nose and Throat 2 (2004): 9-17.

4. Gupta R., et al. "Sinonasal Masses: A Retrospective Analysis". Indian Journal of Otolaryngology and Head and Neck Surgery 65.1 (2013): 52-56.

5. Bhargava R. "A Clinico-Pathological Profile of Sinonasal and Nasopharynx Masses". Journal of Advanced Medical and Dental Sciences Research 8.1 (2020): 218-221.

6. Hedman J., et al. "Prevalence of asthma, aspirin intolerance, nasal polyposis and chronic obstructive pulmonary disease in a population-based study". International Journal of Epidemiology 28.4 (1999): 717-722.

7. Aslıer M and Kasapoğlu F. "Diagnosis Algorithm in Single Sided Nasal Masses". European Journal of Rhinology and Allergy 2.3 (2019): 82-85.

8. Safadi A., et al. "The Efficiency of Routine Histopathological Examination for Bilateral Nasal Polyposis". Ear, Nose and Throat Journal: SAGE Journals (2019): 145561319872728.

9. Zafar U., et al. "Clinicopathological study of non-neoplastic lesions of nasal cavity and paranasal sinuses". Indian Journal of Pathology and Microbiology 51.1 (2008): 26-29.

10. Aljafar HM., et al. "The clinicopathological and radiological features of unilateral nasal mass in adults, a tertiary hospital experience". International Journal of Otorhinolaryngology and Head and Neck Surgery 6 (2020): 1226-1231.

11. Kumar A., et al. "A Clinicopathological Observational Study of Various Types of Masses at Nasal Cavity, Paranasal Sinuses and Nasopharynx". Annals of International Medical and Dental Research 2.6 (2016): EN13-EN15.

12. Ajiya A., et al. "Clinicopathologic Profile of Sinonasal Neoplasia in Kano, Northwestern Nigeria: A 10-Year Single-Institution Experience". Annals of African Medicine 19.3 (2020): 191-197.

13. Agarwal P and Panigrahi R. "Sinonasal Mass-a Recent Study of Its Clinicopathological Profile". The Indian Journal of Surgical Oncology 8.2 (2017): 123-127.

14. Rahghunathan V., et al. "Mucormycosis- can the diagnosis be challenging at times?" SRM Journal of Research in Dental Sciences 9 (2018): 191-196.

15. Spellberg B., et al. "Novel perspectives on mucormycosis: pathophysiology, presentation, and management". Clinical Microbiology Reviews 18.3 (2005): 556-569.
16. Morelli L., et al. "Human nasal rhinosporidiosis: an Italian case report". Diagnostic Pathology 1 (2006): 25.

17. Pradhananga RB., et al. "Overview of nasal masses". Journal of the Institute of Medicine 30 (2008): 13-16.

\section{Volume 3 Issue 9 September 2021}

(C) All rights are reserved by Sahil Kapoor., et al. 